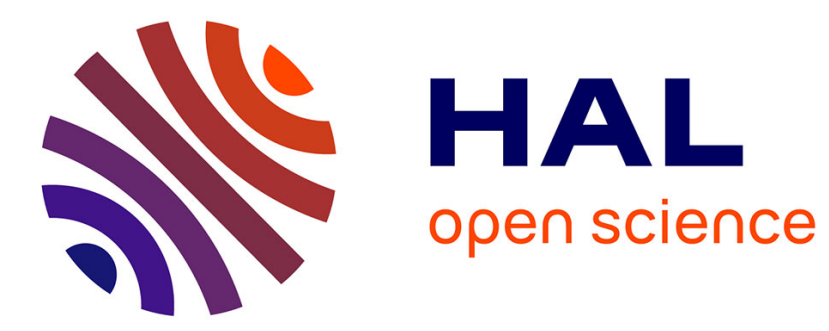

\title{
Inheritance of pollen enzymes and polyploïd origin of apple (Malus x domestica Borkh.)
}

\author{
Yves Lespinasse, Elisabeth Chevreau, M. Gallet
}

\section{To cite this version:}

Yves Lespinasse, Elisabeth Chevreau, M. Gallet. Inheritance of pollen enzymes and polyploïd origin of apple (Malus x domestica Borkh.). TAG Theoretical and Applied Genetics, 1985, 71, pp.268-277. hal-01601149

\section{HAL Id: hal-01601149 \\ https://hal.science/hal-01601149}

Submitted on 2 Jun 2020

HAL is a multi-disciplinary open access archive for the deposit and dissemination of scientific research documents, whether they are published or not. The documents may come from teaching and research institutions in France or abroad, or from public or private research centers.
L'archive ouverte pluridisciplinaire HAL, est destinée au dépôt et à la diffusion de documents scientifiques de niveau recherche, publiés ou non, émanant des établissements d'enseignement et de recherche français ou étrangers, des laboratoires publics ou privés.

\section{다(1)(2)}

Distributed under a Creative Commons Attribution - ShareAlikel 4.0 International 


\title{
Inheritance of pollen enzymes and polyploid origin of apple (Malus $\times$ domestica Borkh.) *
}

\author{
E. Chevreau, Y.Lespinasse and M. Gallet \\ Institut National de la Recherche Agronomique, Station d'Arboriculture Fruitière, Beaucouzé, F-49000 Angers, France
}

Received March 5, 1985; Accepted July 24, 1985

Communicated by F. Salamini

Summary. Electrophoresis of 7 pollen enzymes was applied to 5 progenies from controlled crosses and one self-progeny of apple. Segregation data were examined according to three kinds of hypotheses: monogenic disomic, bigenic disomic and tetrasomic inheritance Twenty codominant alleles and a recessive null were identified. Results provided evidence of bigenic disomic inheritance in most cases: 6 pairs of homoeologous loci carrying identical homoeoalleles were revealed; only 2 enzymes exhibited a simple monogenic control. Preferential pairing between pairs of homologous chromosomes in meiosis can be postulated. These results indicated an allopolyploid origin of apple genome. Fixed heterozygosity occurred for several enzymes, which is a typical feature of allopolyploidy. Loss of duplicate gene expression can account for the monogenic control of 2 of the enzymes.

Key words: Isozymes - Genetic analysis - Polyploidy Malus - Evolution

\section{Introduction}

The cultivated apple (Malus $\times$ domestica Borkh.) belongs to the subfamily Maloideae, formerly known as Pomoideae (Challice 1981). Among the family Rosaceae, the Maloideae raise an interesting evolutionary problem: their basic chromosome number is $x=17$, instead of $x=7,8$ or 9 as in the other subfamilies. Cytological observations reveal a diploid meiotic behaviour: only bivalents can be seen (Lespinasse 1973). An ancient

* The results reported in this paper are part of a thesis by the first author for the degree of "Docteur Ingénieur" polyploid origin is generally assumed, but conflicting hypotheses exist. Darlington and Moffett (1930) postulated an autopolyploid origin supported by cytological observations. According to them, secondary associations of bivalents at meiosis reveal a basic number of $x=7$, suggesting a doubling of the entire set plus the later addition of 3 chromosomes through misdivisions. A basic number of $\mathrm{x}=8$ was also proposed by Sax (1931). Nevertheless, the autopolyploid theory was contested by Sax himself (1933) when he postulated an allopolyploid origin of the Maloideae. Hybridization between primitive members of the sub-families Spiraeoideae $(\mathrm{x}=9)$ and Prunoideae $(\mathrm{x}=8)$ was suggested by Stebbins (1950), on morphological grounds. More recent biochemical studies have supported this hypothesis (Challice 1981).

Different meanings have been given to the concepts of autopolyploidy and allopolyploidy depending on the point of view: cytological, taxonomical or genetical. They appear rather inadequate to classify most natural polyploids (De Wet 1980). According to Sanford (1983), it would be better to use the terms polysomic and disomic, because of their accurate definition: polysomic refers to homologous genomes with random chromosome pairing in meiosis, while disomic refers to homoeologous genomes with preferential pairing between homologous chromosomes in meiosis. The genetical consequences are either polysomic or disomic segregation patterns.

Electrophoretic variants are usually specified by codominant alleles at one or several loci. Isozyme inheritance has been studied in different plant species to obtain evidence of polysomic versus disomic polyploidy. Tetrasomic inheritance at three isozymic loci was found in alfalfa by Quiros (1982) where tetra-allelic genotypes and phenotypic classes resulting from double-reduction were observed. Lewis et al. (1980), studying phosphoglucoisomerase diversity in hexaploid Festuca 
arundinacea, obtained evidence of disomic inheritance indicating preferential pairing between homologous chromosomes. A similar result was reported by Hutchinson et al. (1983) in Avena barbata: six pairs of homoeologous enzyme loci, carrying "homoeoalleles" proved to have a disomic inheritance, as expected in a diploidized tetraploid. Very few isozyme studies have been reported in apple, and of those, most are dealing with cultivar identification (Vinterhalter and James 1982/83) or the relationship between agronomical characters and isozyme patterns (Zhang et al. 1983). To our knowledge, there has only been one attempt to elucidate the genetic control of apple enzymes: Misic etal. (1981) demonstrated disomic inheritance for some bands of bark peroxidase zymograms.

The present study was designed to examine the inheritance of 7 pollen enzymes in apple. Each observed segregation was tested for goodness of fit with three hypotheses: monogenic disomic, bigenic disomic and tetrasomic inheritance. Results are discussed in relation to the different possible origins of the apple genome.

\section{Material and methods}

Pollen was collected from flowers prior to their opening and stored in a dry atmosphere at $-20^{\circ} \mathrm{C}$. A quick rinse with pentane was applied to separate the pollen from the anthers (Cadic 1982). Extracts were prepared by homogeneization of $100 \mathrm{mg}$ pollen in $0.5 \mathrm{ml}$ Tris $\mathrm{KCl} 0.1 \mathrm{M} \mathrm{pH}=7$ buffer, followed by centrifugation. Electrophoresis was performed in polyacrylamide gels for the enzymes esterase (EST), acid phosphatase (AP), endopeptidase (ENP) and in horizontal starch gel for alcohol dehydrogenase $(\mathrm{ADH})$, isocitrate dehydrogenase (IDH), phosphoglucomutase (PGM) and phosphoglucoisomerase (PGI). Gel compositions, running conditions and staining solutions are described in Chevreau (1984).

Five progenies from controlled crosses and one selfprogeny were analysed. Most of them are from breeding programmes. Two progenies had been subjected to a selection on adult phase for tree and fruit characters:

- 'Golden Delicious' (G.) × 'Reinette Clochard' (R.C.).

33 plants analysed.

- 'Golden Delicious' (G.) × 'Delicious' (D.), 22 plants analysed. Five mutants from the same genotype 'Delicious' were used; they proved to have identical zymograms.

Two progenies had been selected only for scab and mildew resistance:

- 'Florina' (F.) × 'Prima' (P.), 40 plants analysed.

- P5 R42 A52 (P5.)× ‘Granny Smith' (G.S.), 41 plants analysed. Two progenies had not been selected:

- 'Delicious' (D.) $\times$ T.N.R., 60 plants analysed. T.N.R. is homozygous for a dominant marker gene coding for high anthocyanin content in all organs.

- self-progeny from P7 R34 A20 (P7.), 37 plants analysed. P7 R34 A20 has been obtained from selfing of the variety 'Golden Delicious'.

Some genetical information were obtained from the analysis of a varietal collection: these results are mentioned when they provide helpful arguments in the discussion.

Three different hypotheses were tested to interpret each enzyme segregation:

- monogenic disomic inheritance refers to the behaviour of a pure diploid species.
- bigenic disomic inheritance supposes the existence of a pair of homoeologous loci segregating independently. When it was possible, two cases were considered: whether the two loci carry identical homoeoalleles, or specific ones. According to Hutchinson (1983), the same symbol is used to designate both homoeologous loci.

- tetrasomic inheritance is assumed when four homologous chromosomes pair at random at meiosis. In the case of apple, only bivalents are formed, therefore no double reduction events are expected. Chromosomal segregation is postulated.

\section{Results}

\section{Endopeptidase}

Only two bands can be seen in endopeptidases zymograms (Fig. 1), although they are often accompanied by a very faint faster band. Alleles associated with the main bands were named $E n p^{a}$ and $E n p^{b}$. The results are shown in Table 1.

Monogenic disomic inheritance does not fit the observed patterns, since absence of segregation in F. $\times$ P. and D. $\times$ T.N.R. progenies cannot be explained. Furthermore, a phenotypic class [b] would be expected in P5. $\times$ G.S. progeny.

Bigenic disomic inheritance is consistent with the observed segregations. As the band a is always present. we can assume that one locus of the pair is fixed for the allele Enp ${ }^{a}$. Depending on the homoeology of the two loci, two cases are considered: the second locus can carry the alleles $E n d^{a}$ and $E n d^{b}$ or it can carry only the $E n d^{b}$ allele plus a null one $E n d^{n}$. A tetrasomic inheritance model is also consistent with the observed segregations.

Table 1 shows that the expected frequencies are the same according to the different hypotheses, and that they fit the observed ratios correctly except in the G. $\times D$. cross; in this progeny, the only plant with the phenotype [a] can be either an accidental out-cross or a self offspring.

\section{Acid phosphatase}

Acid phosphatase zymograms comprise several regions; only the most distant from the origin is interpreted. In this zone, two main band positions, governed by the alleles $A p^{a}$ and $A p^{b}$, can be identified (Fig. 1). Faint closely adjacent bands can also be observed: we consider them to be secondary isozymes. Results are shown in Table 2.

As for the locus Enp, monogenic disomic inheritance is not compatible with our results, since absence of segregation in the self progeny and the cross G. $\times$ R.C. would not be explained. Bigenic disomic inheritance can account for the observed phenotypes in progenies. Again, two cases were distinguished: in the first one, both loci carry the same alleles $A p^{a}$ and $A p^{b}$; in the 

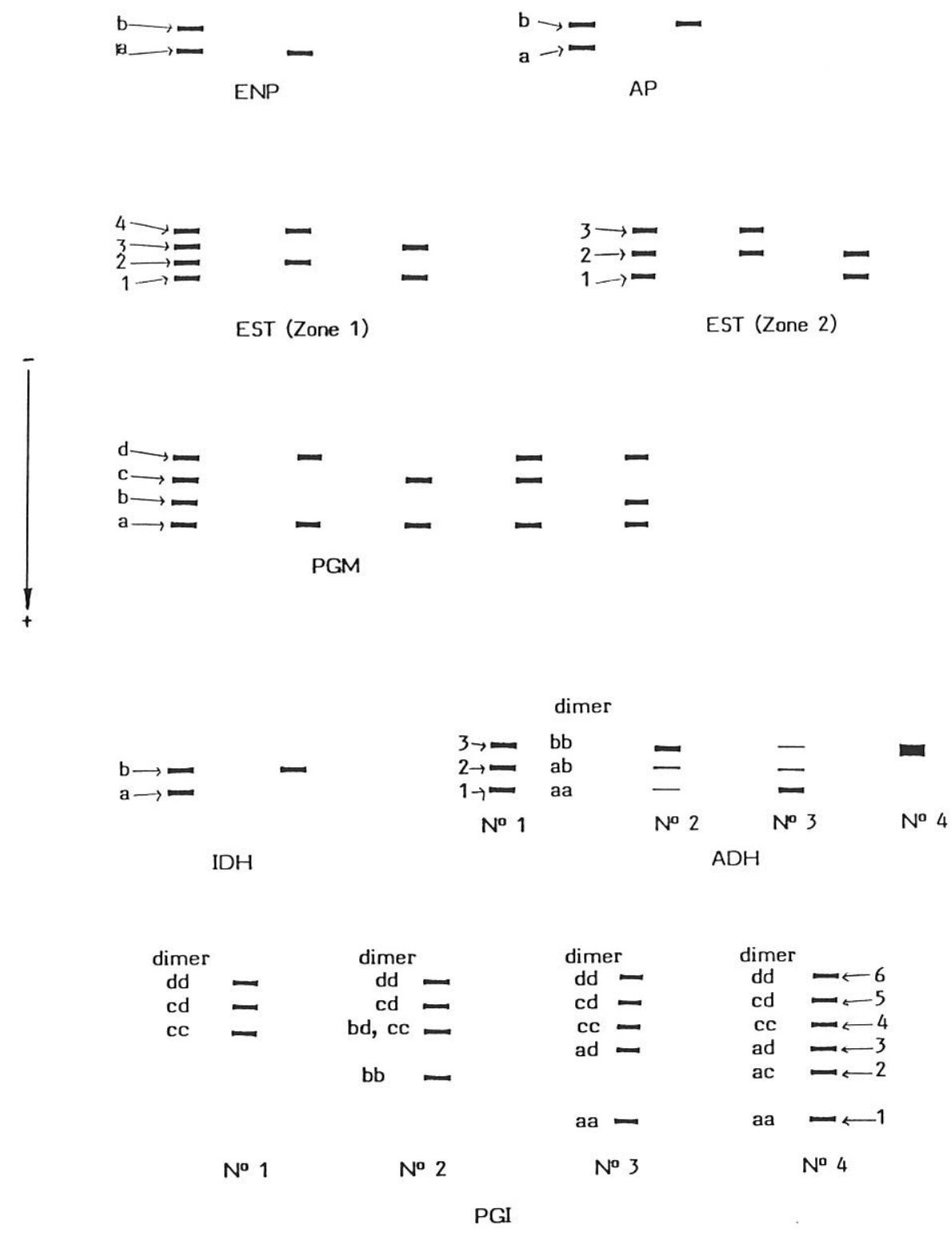

Fig. 1. Schematic representation of the observed phenotypes: for the enzymes ENP, AP, PGM and IDH, letters indicate the band positions. For EST phenotypes. figures are used instead of letters because each pair of bands is governed by one allele, designated by a letter. For $\mathrm{ADH}$ and $\mathrm{PGI}$ phenotypes, figures indicate the band positions and letters the dimeric structure of each band

second case, one locus is always homozygous for $A p^{b}$ and the other one carries $A p^{a}$ or a null allele $A p^{n}$. With respect to the expected frequencies, only one difference can be seen: in the G. $\times$ D. progeny, the first hypothesis fits the observed ratio best.

Tetrasomic inheritance is also consistent with the observed segregations. In the self-progeny, expected frequencies of $1 / 36$ of the homozygous phenotypes may not have been detected because of the small size of the sample.

One progeny deviates significantly from the expected ratio: in the cross D. $\times$ T.N.R., the lower than expected fraction of heterozygous phenotypes is difficult to explain. The male parent T.N.R. carries a homozygous dominant marker gene coding for the red colour of all organs; the whole analysed progeny was red, thus confirming its hybrid origin. Furthermore, no conscious selection has been applied to it. The observed deviation could be explained by gametic selection if this phenomenon was controlled by a locus closely linked with the Ap locus.

\section{Esterase}

Esterase zymograms are complex. Only two zones are interpreted (Figs. 1 and 2): four bands can be seen in the faster zone (zone 1), and three bands in the adjacent one (zone 2). Bands of the two zones are supposed to be controlled by two different loci: Est 1 and Est 2 . 
Table 1. Segregation data and genetic interpretations for locus Enp

\begin{tabular}{|c|c|c|c|c|c|c|c|}
\hline \multirow{2}{*}{\multicolumn{2}{|c|}{$\begin{array}{l}\text { Parental } \\
\text { phenotypes }\end{array}$}} & \multicolumn{3}{|c|}{ Phenotypes in progeny } & \multicolumn{2}{|c|}{ Parental genotypes } & \multirow[t]{3}{*}{$\chi^{2}$} \\
\hline & & \multirow{2}{*}{ [a] } & \multirow[t]{2}{*}[ab]{} & \multirow[t]{2}{*}{ [b] } & \multirow{2}{*}{$q$} & \multirow[t]{2}{*}{$\sigma$} & \\
\hline q & o & & & & & & \\
\hline $\begin{array}{l}\text { G. } \\
{[a]}\end{array}$ & $\times \underset{[a b]}{\text { R.C. }}$ & $\begin{array}{l}15 \\
15.5 \\
15.5 \\
15.5\end{array}$ & $\begin{array}{l}16 \\
15.5 \\
15.5 \\
15.5\end{array}$ & $\begin{array}{l}- \\
- \\
- \\
-\end{array}$ & $\begin{array}{l}\text { (1) aa, aa } \\
\text { (2) aa, nn } \\
\text { (3) }(\mathrm{a})^{4}\end{array}$ & $\begin{array}{l}\text { aa, ab } \\
\text { aa, bn } \\
(\mathrm{a})^{3} \text { b }\end{array}$ & $\begin{array}{l}0.03 \\
0.03 \\
0.03\end{array}$ \\
\hline $\begin{array}{l}\text { G. } \\
{[a]}\end{array}$ & $\times \underset{[a b]}{D}$ & $\begin{array}{l}1 \\
- \\
- \\
-\end{array}$ & $\begin{array}{l}21 \\
22 \\
22 \\
22\end{array}$ & $\begin{array}{l}- \\
- \\
- \\
-\end{array}$ & $\begin{array}{l}\text { (1) aa, aa } \\
\text { (2) aa, nn } \\
\text { (3) (a) }{ }^{4}\end{array}$ & $\begin{array}{l}a a, b b \\
a a, b b \\
a(b)^{3}\end{array}$ & $\begin{array}{l}- \\
- \\
-\end{array}$ \\
\hline $\begin{array}{l}\text { F. } \\
{[a b]}\end{array}$ & $\times \begin{array}{l}\mathrm{P} . \\
{[\mathrm{a}]}\end{array}$ & $\begin{array}{l}- \\
- \\
- \\
-\end{array}$ & $\begin{array}{l}36 \\
36 \\
36 \\
36\end{array}$ & $\begin{array}{l}- \\
- \\
- \\
-\end{array}$ & $\begin{array}{l}\text { (1) aa, bb } \\
\text { (2) aa, bb } \\
\text { (3) a (b) }\end{array}$ & $\begin{array}{l}\text { aa, aa } \\
\text { aa, nn } \\
(\text { a })^{4}\end{array}$ & $\begin{array}{l}- \\
- \\
-\end{array}$ \\
\hline $\begin{array}{l}\text { D. } \\
{[\mathrm{ab}]}\end{array}$ & $\begin{array}{c}\times \text { T.N.R. } \\
{[\mathrm{ab}]}\end{array}$ & $\begin{array}{l}- \\
- \\
- \\
-\end{array}$ & $\begin{array}{l}55 \\
55 \\
55 \\
55\end{array}$ & $\begin{array}{l}- \\
- \\
- \\
-\end{array}$ & $\begin{array}{l}\text { (1) aa, bb } \\
\text { (2) aa, bb } \\
\text { (3) a (b) }\end{array}$ & $\begin{array}{l}a a, b b \\
a a, b b \\
(a)^{3} b\end{array}$ & $\begin{array}{l}- \\
- \\
-\end{array}$ \\
\hline $\begin{array}{l}\text { P5. } \\
\text { [ab] }\end{array}$ & $\times \underset{[a b]}{G . S}$ & $\begin{array}{c}12 \\
9.5 \\
9.5 \\
9.5\end{array}$ & $\begin{array}{l}26 \\
28.5 \\
28.5 \\
28.5\end{array}$ & $\begin{array}{l}- \\
- \\
- \\
-\end{array}$ & $\begin{array}{l}\text { (1) aa, ab } \\
\text { (2) aa, bn } \\
\text { (3) }(a)^{3} b\end{array}$ & $\begin{array}{l}a a, a b \\
a a, b n \\
(a)^{3} b\end{array}$ & $\begin{array}{l}0.87 \\
0.87 \\
0.87\end{array}$ \\
\hline $\begin{array}{l}\text { P7. (s } \\
\text { [a] }\end{array}$ & lfing) & $\begin{array}{l}29 \\
29 \\
29 \\
29\end{array}$ & $\begin{array}{l}- \\
- \\
- \\
-\end{array}$ & $\begin{array}{l}- \\
- \\
- \\
-\end{array}$ & & $\begin{array}{l}\text { (1) aa, aa } \\
\text { (2) aa, nn } \\
\text { (3) }(a)^{4}\end{array}$ & $\begin{array}{l}- \\
- \\
-\end{array}$ \\
\hline
\end{tabular}

(1) Bigenic disomic inheritance, with identical alleles at both loci

(2) Bigenic disomic inheritance, with specific alleles at each locus

(3) Tetrasomic inheritance

$\mathrm{a}=E n p^{a}, \mathrm{~b}=E n p^{b}, \mathrm{n}=E n p^{n}$

In all Tables the observed frequencies are printed in italics: ${ }^{*}$ Significant $(P<0.05)$; ** Significant $(P<0.01)$

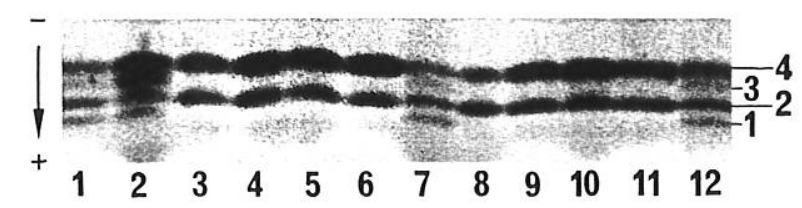

Fig. 2. Esterase zymograms (Zone 1) of the progeny G. $\times$ R.C. Two phenotypes can be seen: [1-2-3-4] in samples 1, 2, 7 and 12 , and $[2-4]$ in the other samples

Zone 1. All the observed phenotypes contain, simultaneously, the bands 2 and 4 , or 1 and 3 . Thus, the existence of two alleles can be postulated: Est $1^{a}$ controlling the double-banded pattern [1-3] and Est $1^{b}$ the pattern [2-4]. Segregation data are reported in Table 3 .
Monogenic disomic inheritance is in agreement with the results; only $1: 1$ or $1: 0$ segregations were observed. Tetrasomic inheritance can also explain these frequencies.

Zone 2. As in zone 1, two alleles controlling doublebanded patterns are proposed: Est $2^{a}$ for phenotype [1-2] and Est $2^{b}$ for phenotype [2-3]. In two progenies, some plants exhibited a "null" phenotype; so, a null allele Est $2^{n}$ was also postulated. Results are shown in Table 4. Segregation data from the D. $\times$ T.N.R. progeny are not reported because the male parent phenotype could not be ascertained.

Monogenic disomic inheritance can account for the observed phenotypic classes; but in the P5.×G.S. progeny, observed frequencies deviate significantly from the expected ones. 
Table 2. Segregation data and genetic interpretations for locus $A p$

\begin{tabular}{|c|c|c|c|c|c|c|c|}
\hline \multirow{2}{*}{\multicolumn{2}{|c|}{$\begin{array}{l}\text { Parental } \\
\text { phenotypes }\end{array}$}} & \multicolumn{3}{|c|}{ Phenotypes in progeny } & \multicolumn{2}{|c|}{ Parental genotypes } & \multirow[t]{3}{*}{$\chi^{2}$} \\
\hline & & \multirow[t]{2}{*}{ [a] } & \multirow[t]{2}{*}[ab]{} & \multirow[t]{2}{*}{ [b] } & \multirow[t]{2}{*}{$q$} & \multirow[t]{2}{*}{$\hat{\sigma}$} & \\
\hline$q$ & $\hat{\sigma}$ & & & & & & \\
\hline $\begin{array}{l}\text { F. } \\
\text { [b] }\end{array}$ & $\times \underset{[\mathrm{b}]}{\mathrm{P} .}$ & $\begin{array}{l}- \\
- \\
- \\
-\end{array}$ & $\begin{array}{l}- \\
- \\
- \\
-\end{array}$ & $\begin{array}{l}37 \\
37 \\
37 \\
37\end{array}$ & $\begin{array}{l}\text { (1) bb, bb } \\
\text { (2) nn, bb } \\
\text { (3) (b) }{ }^{4}\end{array}$ & $\begin{array}{l}\mathrm{bb}, \mathrm{bb} \\
\mathrm{nn}, \mathrm{bb} \\
(\mathrm{b})^{4}\end{array}$ & $\begin{array}{l}- \\
- \\
-\end{array}$ \\
\hline $\begin{array}{l}\text { G. } \\
{[a b]}\end{array}$ & $\begin{array}{l}\times \quad \mathrm{D} . \\
{[\mathrm{b}]}\end{array}$ & $\begin{array}{l}- \\
- \\
- \\
-\end{array}$ & $\begin{array}{l}15 \\
16.5 \\
11 \\
11\end{array}$ & $\begin{array}{l}7 \\
5.5 \\
11 \\
11\end{array}$ & $\begin{array}{l}\text { (1) ab, ab } \\
\text { (2) an, bb } \\
\text { (3) a (b) }\end{array}$ & $\begin{array}{l}\mathrm{bb}, \mathrm{bb} \\
\mathrm{nn}, \mathrm{bb} \\
(\mathrm{b})^{4}\end{array}$ & $\begin{array}{l}0.54 \\
2.90 \\
2.90\end{array}$ \\
\hline $\begin{array}{l}\text { P5. } \\
{[\mathrm{b}]}\end{array}$ & $\times \underset{[a b]}{G . S .}$ & $\begin{array}{l}- \\
- \\
- \\
-\end{array}$ & $\begin{array}{l}19 \\
19.5 \\
19.5 \\
19.5\end{array}$ & $\begin{array}{l}20 \\
19.5 \\
19.5 \\
19.5\end{array}$ & $\begin{array}{l}\text { (1) bb, bb } \\
\text { (2) nn, bb } \\
\text { (3) }(b)^{4}\end{array}$ & $\begin{array}{l}a b, b b \\
\text { an, bb } \\
a(b)^{3}\end{array}$ & $\begin{array}{l}0.02 \\
0.02 \\
0.02\end{array}$ \\
\hline $\begin{array}{l}\text { D. } \\
{[\mathrm{b}]}\end{array}$ & $\times \underset{[a b]}{\text { T.N.R. }}$ & $\begin{array}{l}- \\
- \\
- \\
-\end{array}$ & $\begin{array}{r}9 \\
28 \\
28 \\
28\end{array}$ & $\begin{array}{l}47 \\
28 \\
28 \\
28\end{array}$ & $\begin{array}{l}\text { (1) bb. bb } \\
\text { (2) nn. bb, } \\
\text { (3) }(b)^{4}\end{array}$ & $\begin{array}{l}a b, b b \\
a n, b b \\
a(b)^{3}\end{array}$ & $\begin{array}{l}25^{* *} \\
25^{* *} \\
25^{* *}\end{array}$ \\
\hline $\begin{array}{l}\text { G. } \\
{[a b]}\end{array}$ & $\times \underset{[a b]}{\text { R.C. }}$ & $\begin{array}{l}- \\
- \\
- \\
-\end{array}$ & $\begin{array}{l}28 \\
28 \\
28 \\
28\end{array}$ & $\begin{array}{l}- \\
- \\
- \\
-\end{array}$ & $\begin{array}{l}\text { (1) ab. ab } \\
\text { (2) an. bb } \\
\text { (3) a (b) })^{3}\end{array}$ & $\begin{array}{l}a a, b b \\
a a, b b \\
(a)^{3} b\end{array}$ & $\begin{array}{l}- \\
- \\
-\end{array}$ \\
\hline $\begin{array}{l}\text { P7. }(\mathrm{s} \\
{[\mathrm{ab}]}\end{array}$ & ffing) & $\begin{array}{l}- \\
- \\
- \\
0.9\end{array}$ & $\begin{array}{l}31 \\
31 \\
31 \\
29.2\end{array}$ & $\begin{array}{l}- \\
- \\
- \\
0.9\end{array}$ & & $\begin{array}{l}\text { (1) aa, aa } \\
\text { (2) aa, bb } \\
(3)(a)^{2}(b)^{2}\end{array}$ & $\begin{array}{l}- \\
- \\
-\end{array}$ \\
\hline
\end{tabular}

(1) Bigenic disomic inheritance, with identical alleles at both loci

(2) Bigenic disomic inheritance, with specific alleles at each locus

(3) Tetrasomic inheritance

$\mathrm{a}=A p^{a}, \mathrm{~b}=A p^{b}, \mathrm{n}=A p^{n}$

Tetrasomic inheritance can also be used, although goodness-of-fit is very poor in the case of G. $\times$ R.C. progeny.

Bigenic disomic inheritance provides the best interpretation of these results. We assumed that both homoeologous loci can carry identical alleles. In every progeny, observed frequencies fit correctly the expected ratios.

\section{Phosphoglucomutase}

Four main band positions were observed in phosphoglucomutase zymograms (Fig. 1). The corresponding alleles were named $\mathrm{Pgm}^{a}, \mathrm{Pgm}^{b}, \mathrm{Pgm}^{c}$ and $\mathrm{Pgm}^{d}$. Two faster bands appeared sometimes: they were too faint to be taken into account. Table 5 shows the results.

Monogenic disomic interpretation could not be used because of the occurrence of tri- and tetra-allelic geno- types. Neither can tetrasomic inheritance explain th phenotypes in the progenies, since more classes woul be expected in every case. Bigenic disomic inheritanc is the only suitable hypothesis. Two cases are di: tinguished: either both loci carry identical alleles c one of them governs the two faster bands (alleles Pgm and $P g m^{b}$ only) and the other one the two slowe (alleles $\mathrm{Pgm}^{c}$ and $\mathrm{Pgm}^{d}$ only). The goodness-of-fitnes test is in favour of the first hypothesis.

Whatever the interpretaion, observed frequencies $i$ the D. $\times$ T.N.R. progeny deviate significantly from th theoretical ones. Excess of phenotypes with the band can be explained by a gametic selection in favour $c$ pollen grains carrying the allele $\mathrm{Pgm}^{b}$.

In the self progeny, three plants exhibit an unex pected phenotype [acd], band $\mathbf{c}$ is absent in the parenta zymogram. These plants are certainly accidental out crosses; this is confirmed by their IDH zymograms an 
Table 3. Segregation data and genetic interpretations for locus Est 1

\begin{tabular}{|c|c|c|c|c|c|c|c|c|}
\hline \multirow{2}{*}{\multicolumn{3}{|c|}{$\begin{array}{l}\text { Parental } \\
\text { phenotypes }\end{array}$}} & \multicolumn{3}{|c|}{ Phenotypes in progeny } & \multicolumn{2}{|c|}{ Parental genotypes } & \multirow[t]{3}{*}{$\chi^{2}$} \\
\hline & & & \multirow{2}{*}[1-3]{} & \multirow[t]{2}{*}[1-2-3-4]{} & \multirow[t]{2}{*}[2-4]{} & \multirow[t]{2}{*}{$q$} & \multirow[t]{2}{*}{$\delta$} & \\
\hline$q$ & $\sigma^{\hat{\sigma}}$ & & & & & & & \\
\hline $\begin{array}{l}\text { G. } \\
{[2-4]}\end{array}$ & $\times$ & $\begin{array}{l}\text { R.C. } \\
{[1-2-3-4]}\end{array}$ & $\begin{array}{l}- \\
- \\
-\end{array}$ & $\begin{array}{l}17 \\
16.5 \\
16.5\end{array}$ & $\begin{array}{l}16 \\
16.5 \\
16.5\end{array}$ & $\begin{array}{l}\text { (1) bb } \\
\text { (2) }(b)^{4}\end{array}$ & $\begin{array}{l}a b \\
a(b)^{3}\end{array}$ & $\begin{array}{l}0.03 \\
0.03\end{array}$ \\
\hline $\begin{array}{l}\text { D. } \\
{[2-4]}\end{array}$ & $\times$ & $\begin{array}{l}\text { T.N.R. } \\
{[1-2-3-4]}\end{array}$ & - & $\begin{array}{l}33 \\
30 \\
30\end{array}$ & $\begin{array}{l}27 \\
30 \\
30\end{array}$ & $\begin{array}{l}\text { (1) bb } \\
\text { (2) }(b)^{4}\end{array}$ & $\begin{array}{l}a b \\
a(b)^{3}\end{array}$ & $\begin{array}{l}0.60 \\
0.60\end{array}$ \\
\hline $\begin{array}{l}\text { P.5 } \\
{[2-4]}\end{array}$ & & $\begin{array}{l}\text { G.S. } \\
{[1-3]}\end{array}$ & $\begin{array}{l}- \\
- \\
-\end{array}$ & $\begin{array}{l}41 \\
41 \\
41\end{array}$ & $\begin{array}{l}- \\
- \\
-\end{array}$ & $\begin{array}{l}\text { (1) bb } \\
\text { (2) (b) }\end{array}$ & $\begin{array}{l}\text { aa } \\
(\mathrm{a})^{4}\end{array}$ & $\begin{array}{l}- \\
-\end{array}$ \\
\hline $\begin{array}{l}\text { F. } \\
{[2-4]}\end{array}$ & $\times$ & $\begin{array}{l}\text { P. } \\
{[2-4]}\end{array}$ & $\begin{array}{l}- \\
- \\
-\end{array}$ & $\begin{array}{l}- \\
- \\
-\end{array}$ & $\begin{array}{l}38 \\
38 \\
38\end{array}$ & $\begin{array}{l}\text { (1) bb } \\
\text { (2) }(b)^{4}\end{array}$ & $\begin{array}{l}\text { bb } \\
(b)^{4}\end{array}$ & $\begin{array}{l}- \\
-\end{array}$ \\
\hline $\begin{array}{l}\text { G. } \\
{[2-4]}\end{array}$ & & $\begin{array}{l}\text { D. } \\
{[2-4]}\end{array}$ & $\begin{array}{l}- \\
- \\
-\end{array}$ & $\begin{array}{l}- \\
- \\
-\end{array}$ & $\begin{array}{l}22 \\
22 \\
22\end{array}$ & $\begin{array}{l}\text { (1) bb } \\
\text { (2) }(b)^{4}\end{array}$ & $\begin{array}{l}\mathrm{bb} \\
(\mathrm{b})^{4}\end{array}$ & - \\
\hline $\begin{array}{l}\text { P7. (se } \\
{[2-4]}\end{array}$ & ing) & & $\begin{array}{l}- \\
- \\
-\end{array}$ & $\begin{array}{l}- \\
- \\
-\end{array}$ & $\begin{array}{l}29 \\
29 \\
29\end{array}$ & & $\begin{array}{l}b b \\
(b)^{4}\end{array}$ & \\
\hline
\end{tabular}

(1) Monogenic disomic inheritance

(2) Tetrasomic inheritance

$\mathrm{a}=$ Est $1^{a}, \mathrm{~b}=$ Est $1^{b}$

by their unexpected vigor, as opposed to the "inbreeding effect" of the whole self-progeny. Because of their hybrid origin, these 3 plants were eliminated from the previous result tables.

\section{Isocitrate dehydrogenase}

Isocitrate dehydrogenase zymograms contain many closely adjacent bands. Only the two faster bands are clearly separated (Figs. 1 and 3); they are interpreted as the products of two alleles $I d h^{a}$ and $I d h^{b}$. Results are presented in Table 6.

Two plants with phenotype [ab] appeared in the self-progeny of a [b] parent; they belong to the three out-crosses described earlier.

Three hypotheses can be used to interpret the data: monogenic disomic inheritance, bigenic disomic inheritance with both loci carrying the alleles $I d h^{a}$ and $I d h^{b}$, and tetrasomic inheritance. The only difference between the expected frequencies appears in the progeny G. $\times$ R.C. In this segregation, the lower than expected fraction of homozygous phenotypes fits better the second or the third interpretation.

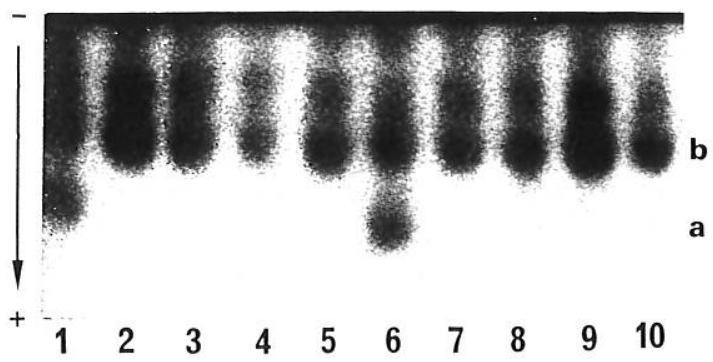

Fig. 3. Isocitrate dehydrogenase zymograms of the self progeny. Samples 1 and 6 exhibit a phenotype $[a-b]$ indicating an out-cross origin

Another observation must be considered: in many plant species, IDH proved to be a dimeric enzyme. Interesting information about the quaternary structure of apple IDH were obtained through the analysis of a varietal collection: among 50 varieties, four exhibited an additional IDH band between the bands $\mathbf{a}$ and $\mathbf{b}$. These varieties were three spontaneous tetraploid mutants from diploid varieties having the usual pheno- 
Table 4. Segregation data and genetic interpretations for locus Est 2

\begin{tabular}{|c|c|c|c|c|c|c|c|c|c|}
\hline \multirow{2}{*}{\multicolumn{3}{|c|}{$\begin{array}{l}\text { Parental } \\
\text { phenotypes }\end{array}$}} & \multicolumn{3}{|c|}{ Phenotypes in progeny } & \multirow{3}{*}[\quad]{} & \multicolumn{2}{|c|}{ Parental genotypes } & \multirow[t]{3}{*}{$\chi^{2}$} \\
\hline & & & \multirow{2}{*}[1-2]{} & \multirow{2}{*}[1-2-3]{} & \multirow{2}{*}[2-3]{} & & \multirow[t]{2}{*}{$q$} & \multirow[t]{2}{*}{$\delta$} & \\
\hline$q$ & $\hat{\sigma}$ & & & & & & & & \\
\hline $\begin{array}{l}\text { F. } \\
{[1-2-3]}\end{array}$ & $\times$ & $\begin{array}{l}\mathrm{P} . \\
{[2-3]}\end{array}$ & $\begin{array}{l}- \\
- \\
- \\
-\end{array}$ & $\begin{array}{l}24 \\
18.5 \\
27.75 \\
18.5\end{array}$ & $\begin{array}{l}13 \\
18.5 \\
9.25 \\
18.5\end{array}$ & $\begin{array}{l}- \\
- \\
- \\
-\end{array}$ & $\begin{array}{l}\text { (1) ab } \\
\text { (2) ab, ab } \\
\text { (3) a (b) }\end{array}$ & $\begin{array}{l}\text { bb } \\
b b, b b \\
(b)^{4}\end{array}$ & $\begin{array}{l}3.27 \\
2.02 \\
3.27\end{array}$ \\
\hline $\begin{array}{l}\text { P5. } \\
{[1-2-3]}\end{array}$ & & $\begin{array}{l}\text { G.S. } \\
{[2-3]}\end{array}$ & $\begin{array}{l}8 \\
10.25 \\
5.125 \\
3.43\end{array}$ & $\begin{array}{l}15 \\
10.25 \\
15.375 \\
17.07\end{array}$ & $\begin{array}{l}18 \\
20.5 \\
20.5 \\
20.5\end{array}$ & $\begin{array}{l}- \\
- \\
- \\
-\end{array}$ & $\begin{array}{l}\text { (1) } a b \\
\text { (2) ab, nn } \\
\text { (3) a (b) }{ }^{2} n\end{array}$ & $\begin{array}{l}\text { bn } \\
b n, b n \\
b(n)^{3}\end{array}$ & $\begin{array}{c}18.8^{* *} \\
1.92 \\
6.64^{*}\end{array}$ \\
\hline $\begin{array}{l}\text { G. } \\
{[2-3]}\end{array}$ & $\times$ & $\begin{array}{l}\text { R.C. } \\
{[1-2-3]}\end{array}$ & $\begin{array}{l}6 \\
8.25 \\
8.25 \\
2.75\end{array}$ & \begin{tabular}{r}
\multicolumn{1}{l}{5} \\
8.25 \\
8.25 \\
24.75
\end{tabular} & $\begin{array}{l}22 \\
16.5 \\
16.5 \\
5.5\end{array}$ & $\begin{array}{l}- \\
- \\
- \\
-\end{array}$ & $\begin{array}{l}\text { (1) bn } \\
\text { (2) bn. nn } \\
\text { (3) b (n) }\end{array}$ & $\begin{array}{l}\mathrm{ab} \\
\mathrm{ab}, \mathrm{nn} \\
(\mathrm{a})^{2}(\mathrm{~b})^{2}\end{array}$ & $\begin{array}{r}3.72 \\
3.72 \\
69^{* *}\end{array}$ \\
\hline $\begin{array}{l}\text { G. } \\
{[2-3]}\end{array}$ & $\times$ & $\begin{array}{l}\text { D. } \\
{[2-3]}\end{array}$ & $\begin{array}{l}- \\
- \\
- \\
-\end{array}$ & $\begin{array}{l}- \\
- \\
- \\
-\end{array}$ & $\begin{array}{l}20 \\
16.5 \\
19.25 \\
20.17\end{array}$ & $\begin{array}{l}2 \\
5.5 \\
2.75 \\
1.83\end{array}$ & $\begin{array}{l}\text { (1) bn } \\
\text { (2) bn, nn } \\
\text { (3) b (n) }\end{array}$ & $\begin{array}{l}\text { bn } \\
\text { bn, bn } \\
(b)^{2}(n)^{2}\end{array}$ & $\begin{array}{l}2.96 \\
0.23 \\
0.02\end{array}$ \\
\hline $\begin{array}{l}\text { P7. (self } \\
{[2-3]}\end{array}$ & ing) & & $\begin{array}{l}- \\
- \\
- \\
-\end{array}$ & $\begin{array}{l}- \\
- \\
- \\
-\end{array}$ & $\begin{array}{l}26 \\
21.75 \\
27.19 \\
21.75\end{array}$ & $\begin{array}{l}3 \\
7.25 \\
1.81 \\
7.25\end{array}$ & & $\begin{array}{l}\text { (1) bn } \\
\text { (2) bn, bn } \\
\text { (3) b (n) }\end{array}$ & $\begin{array}{l}3.32 \\
0.83 \\
3.32\end{array}$ \\
\hline
\end{tabular}

(1) Monogenic disomic inheritance

(2) Bigenic disomic inheritance. with identical alleles at both loci

(3) Tetrasomic inheritance

$\mathrm{a}=$ Est $2^{a}, \mathrm{~b}=$ Est $2^{b}, \mathrm{n}=$ Est $2^{n}$

type [ab] ('Jonathan', 'Wealthy', 'Delicious'), and one tetraploid seedling from the triploid variety 'Reinette du Canada'. This fact is in agreement with a dimeric structure of apple IDH; we can assume that the additional band is a heterodimeric molecule which can be synthesized only in the diploid pollen grains of a heterozygous tetraploid clone.

This hybrid band was not observed in the progenies from crosses or selfing. This is a convincing argument in favour of a monogenic disomic inheritance; with bigenic disomic inheritance as with tetrasomic inheritance, presence of the hybrid band would be expected in all heterozygous plants.

\section{Alcohol dehydrogenase}

Three bands can be seen in the ADH zymograms (Figs. 1 and 4). All parents and their progenies have the same three banded phenotype (number 1 in Fig. 1). ADH is reported as a dimeric enzyme in many plant species; we can suppose that apple $\mathrm{ADH}$ also has a dimeric structure. On this assumption, this phenotype is inter-

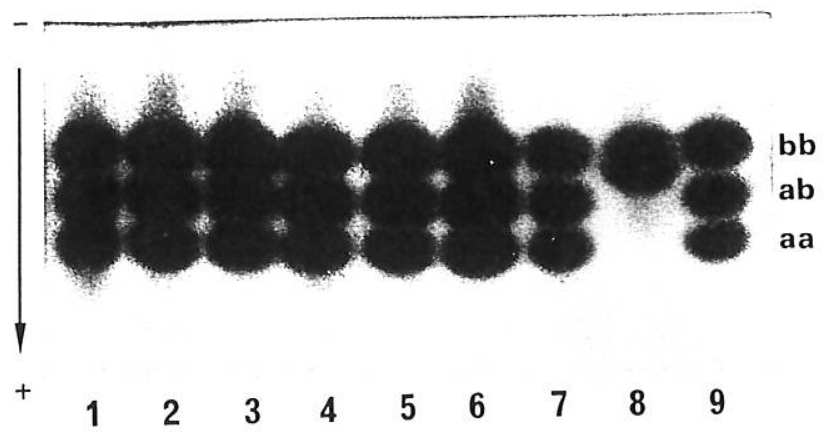

Fig. 4. Alcohol dehydrogenase zymograms of several varieties. Letters refer to the dimeric composition of each band. 'Lodi' (sample 8) is the only variety with a single-banded phenotype

preted as the product of two alleles $A d h^{a}$ and $A d h^{b}$ with the central band corresponding to the heterodimeric molecule.

Monogenic disomic control is incompatible with the appearance of a heterozygous phenotype in pollen: only one allele can be present in each pollen grain, so 
Table 5. Segregation data and genetic interpretations for locus Pgm

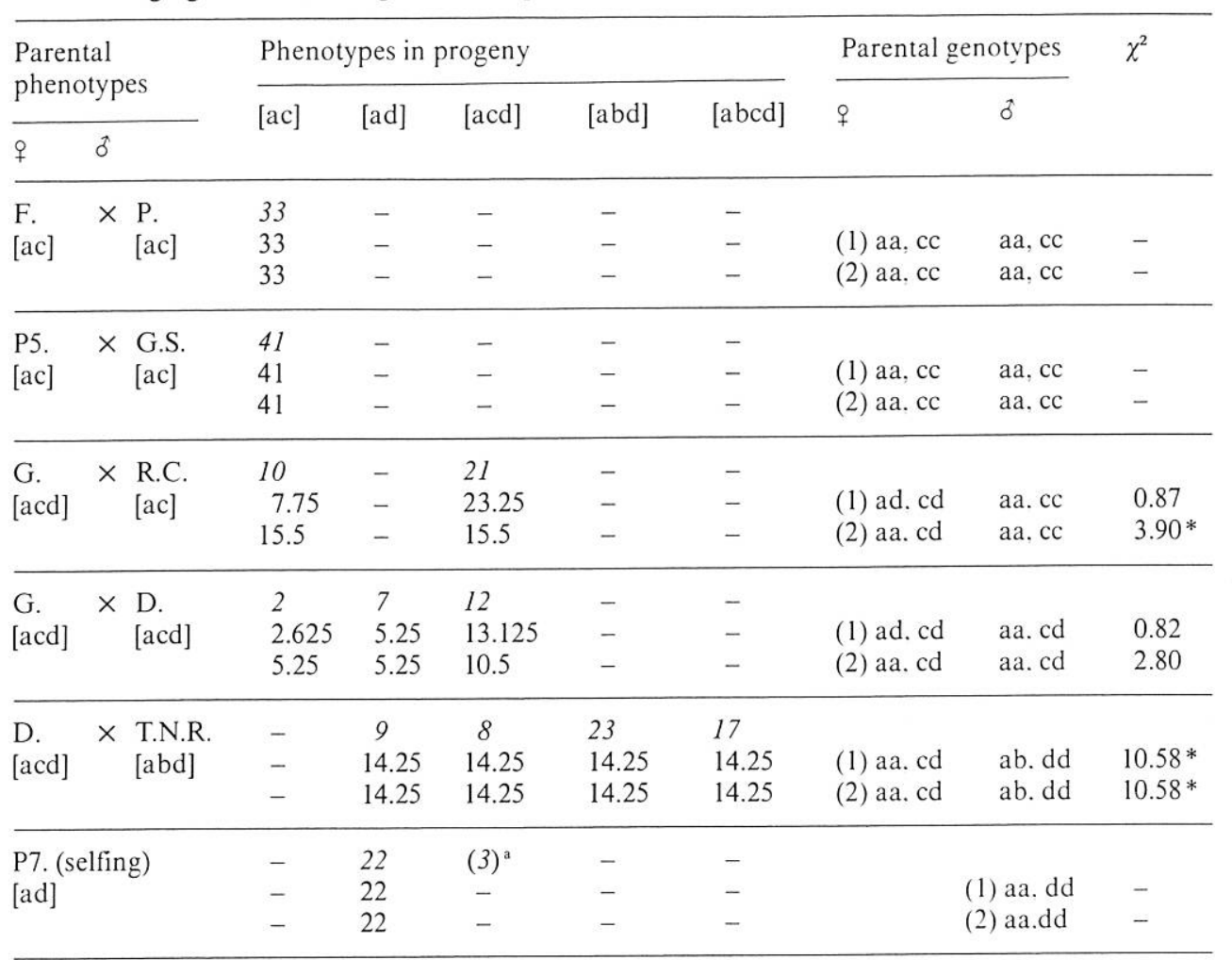

(1) Bigenic disomic inheritance with identical alleles at both loci

(2) Bigenic disomic inheritance with specific alleles at each locus

a These 3 plants proved to be out-crosses

$\mathrm{a}=P g m^{a}, \mathrm{~b}=P g m^{b}, \mathrm{c}=P g m^{c}, \mathrm{~d}=P g m^{d}$

no hybrid molecules should be synthesized. Tetrasomic inheritance cannot account for the absence of segregation in progenies, either by crossing or selfing. Only bigenic disomic inheritance can be responsible for this "fixed heterozygosity": all parents have the same genotype $A d h^{a} A d h^{a}, A d h^{b} A d h^{b}$.

No difference of intensity between the three bands were found in parents and their progeny, although a more intense central band could be expected. Different explanations can be proposed: the association of the subunits is not completely at random, or the activity of the heterodimeric enzyme is less important than the homodimeric one.

Different phenotypes were observed in the varietal collection. Two phenotypes showing intensity variations can be explained by different allelic dosages (Fig. 1): $A d h^{a} A d h^{b}, A d h^{b} A d h^{b}$ for number 2 and $A d h^{a} A d h^{a}$, $A d h^{a} A d h^{b}$ for number 3. Only one variety, 'Lodi', possesses a single banded pattern (number 4), corresponding to the double homozygous genotype: $A d h^{b} A d h^{b}, A d h^{b} A d h^{b}$.

\section{Phosphoglucoisomerase}

Phosphoglucoisomerase patterns are very similar to alcohol dehydrogenase patterns: a three banded phenotype was observed in all parents and in their whole progenies (number 1 in Fig. 1). A dimeric structure of this enzyme can be postulated in reference to reports in other plant species. Thus, this pattern is controlled by two alleles, $P g i^{c}$ and $P g i^{d}$, and the central band is a heterodimeric enzyme.

As for $\mathrm{ADH}$, this true-breeding heterozygous phenotype indicates a bigenic disomic inheritance, with "fixed heterozygosity". No difference of intensity between bands was observed.

The analysis of 50 varieties revealed 3 other phenotypes; they are represented in Fig. 1. The following interpretations can be proposed: in phenotype number 2 . a new band appears, which is controlled by the allele $P g i b$; the heterodimer (bd) cannot be distinguished from the homodimer (cc) and forms band 4. The heterodimer (bc) is not synthesized. This can be explained by the genotype: $P g i^{b} P g i^{c}, P g i^{d} P g i^{d}$ : only 
Table 6. Segregation data and genetic interpretations for locus $I d h$

\begin{tabular}{|c|c|c|c|c|c|c|}
\hline \multicolumn{2}{|c|}{$\begin{array}{l}\text { Parental } \\
\text { phenotypes }\end{array}$} & \multicolumn{2}{|c|}{$\begin{array}{l}\text { Phenotypes } \\
\text { in progeny }\end{array}$} & \multicolumn{2}{|c|}{ Parental genotypes } & \multirow[t]{2}{*}{$\chi^{2}$} \\
\hline$q$ & ot & {$[b]$} & {$[a b]$} & $q$ & o & \\
\hline $\begin{array}{l}\text { F. } \\
{[a b]}\end{array}$ & $\begin{array}{r}\times \\
\\
{[\mathrm{P}]}\end{array}$ & $\begin{array}{l}16 \\
17 \\
17 \\
17\end{array}$ & $\begin{array}{l}18 \\
17 \\
17 \\
17\end{array}$ & $\begin{array}{l}\text { (1) ab } \\
\text { (2) ab, bb } \\
\text { (3) a (b) }\end{array}$ & $\begin{array}{l}\mathrm{bb} \\
\mathrm{bb}, \mathrm{bb} \\
(\mathrm{b})^{4}\end{array}$ & $\begin{array}{l}0.11 \\
0.11 \\
0.11\end{array}$ \\
\hline $\begin{array}{l}\text { D. } \\
{[a b]}\end{array}$ & $\times \underset{\text { T.N.R. }}{\times}$ & $\begin{array}{l}23 \\
21 \\
21 \\
21\end{array}$ & $\begin{array}{l}19 \\
21 \\
21 \\
21\end{array}$ & $\begin{array}{l}\text { (1) } a b \\
\text { (2) } a b, b b \\
\text { (3) } a(b)^{3}\end{array}$ & $\begin{array}{l}\text { bb } \\
\text { bb, bb } \\
(\text { b) })^{4}\end{array}$ & $\begin{array}{l}0.38 \\
0.38 \\
0.38\end{array}$ \\
\hline $\begin{array}{l}\text { G. } \\
{[\mathrm{b}]}\end{array}$ & $\times \underset{[a b]}{D}$ & $\begin{array}{l}11 \\
10.5 \\
10.5 \\
10.5\end{array}$ & $\begin{array}{l}10 \\
10.5 \\
10.5 \\
10.5\end{array}$ & $\begin{array}{l}\text { (1) bb } \\
\text { (2) bb, bb } \\
\text { (3) }(b)^{4}\end{array}$ & $\begin{array}{l}a b \\
a b, b b \\
a(b)^{3}\end{array}$ & $\begin{array}{l}0.04 \\
0.04 \\
0.04\end{array}$ \\
\hline $\begin{array}{l}\text { G. } \\
{[b]}\end{array}$ & $\times \underset{[a b]}{\text { R.C. }}$ & $\begin{array}{c}2 \\
15.5 \\
7.75 \\
5.16\end{array}$ & $\begin{array}{l}29 \\
15.5 \\
23.25 \\
25.84\end{array}$ & $\begin{array}{l}\text { (1) bb } \\
\text { (2) bb, bb } \\
\text { (3) }(b)^{4}\end{array}$ & $\begin{array}{l}a b \\
a b, a b \\
(a)^{2}(b)^{2}\end{array}$ & $\begin{array}{c}23.5^{* *} \\
5.69^{*} \\
2.32\end{array}$ \\
\hline $\begin{array}{l}\text { P5. } \\
{[\mathrm{b}]}\end{array}$ & $\times \underset{[b]}{\text { G.S. }}$ & $\begin{array}{l}40 \\
40 \\
40 \\
40\end{array}$ & $\begin{array}{l}- \\
- \\
- \\
-\end{array}$ & $\begin{array}{l}\text { (1) bb } \\
\text { (2) bb, bb } \\
\text { (3) }(b)^{4}\end{array}$ & $\begin{array}{l}\mathrm{bb} \\
\mathrm{bb}, \mathrm{bb} \\
\text { (b) }\end{array}$ & $\begin{array}{l}- \\
- \\
-\end{array}$ \\
\hline $\begin{array}{l}\text { P7. (se } \\
\text { [b] }\end{array}$ & (selfing) & $\begin{array}{l}21 \\
21 \\
21 \\
21\end{array}$ & $\begin{array}{l}(2)^{a} \\
- \\
- \\
-\end{array}$ & & $\begin{array}{l}b b \\
b b, b b \\
(b)^{4}\end{array}$ & $\begin{array}{l}- \\
- \\
-\end{array}$ \\
\hline
\end{tabular}

(1) Monogenic disomic inheritance

(2) Bigenic disomic inheritance with identical alleles at both loci

(3) Tetrasomic inheritance

a These 2 plants proved to be out-crosses $\mathrm{a}=I d h^{a}, \mathrm{~b}=I d h^{b^{\mathrm{P}}}$

pollen grains carrying together $P g i^{b}$ and $P g i^{d}$ or $P g i^{c}$ and $P g i^{d}$ are formed. The phenotype number 3 is explained in the same way by the genotype: $\mathrm{Pgi}^{a} \mathrm{Pgi}^{\mathrm{c}}$, $P g i{ }^{d} P i^{d}$. In phenotype number 4, three alleles are involved: $P i^{a}, P g i^{c}, P g i^{d}$, and all the possible heterodimers are formed; different genotypes can be proposed, for example: $\mathrm{Pgi}^{a} \mathrm{Pgi}^{c}, \mathrm{Pgi}^{\mathrm{c}} \mathrm{Pgi}^{\mathrm{d}}$.

\section{Discussion}

Inheritance studies in apple are still limited. Maintenance of large unselected progenies until the adult stage is often impossible in the context of a breeding programme. Such difficulties have been encountered during this study because electrophoresis was applied to pollen extracts. Pollen was chosen for several reasons: its enzymatic composition tends to be independent from environmental conditions, extracts are easily prepared because of the absence of polyphenoloxidase, and enzymatic activity in the pollen is important for many different enzymes. However, most available adult progenies were reduced to a selected sample. Our results show three significative deviations from expected frequencies; only one of them appeared in a selected progeny (G. $\times$ R.C., for IDH). In this case, a linkage between one of the selected characters and the locus IDH could be postulated, because this progeny has been subjected to a very high selection pressure. The two other deviations occurred in the progeny D. $\times$ T.N.R., for the enzymes AP and PGM. The marker gene carried by the male parent provides evidence of the hybrid origin of all the plants; furthermore, no selection has been applied to this progeny. Gametophytic selection can often account for deviations in segregation ratios. This has been reviewed recently by Zamir (1983): numerous reports demonstrate pollen gene expression and selection. According to Mulcahy (1979), microgametophytic competition is as a selective mechanism of great importance regarding angiosperms evolution. Such a phenomenon can be postulated here, to explain the deviations observed in the D. $\times$ T.N.R. progeny.

The results reported above lead to several conclusions concerning the inheritance pattern of seven pollen enzymes:

- monogenic disomic inheritance was the simplest hypothesis. However, it cannot be used to explain the data obtained for ENP, AP, PGM, ADH and PGI. Only EST 1 and IDH segregations are consistent with this interpretation.

- tetrasomic inheritance cannot be postulated for PGM, ADH and PGI. In the other cases, this hypothesis is not incompatible with the results, but goodness-of-fit tests are not as good as in the case of bigenic disomic hypothesis. Furthermore, no typical feature of tetrasomic inheritance was observed: phenotypic classes in progenies are always in small number, and examples of trior tetra-allelism at a single locus cannot be found. No evidence of double-reduction events was obtained, which is in agreement with the absence of multivalent pairing at meiosis.

- bigenic disomic inheritance seems to be the best interpretation for most results, with the exception of EST 1 and IDH. It is the only hypothesis consistent with the data of PGM, ADH and PGI. As far as possible, we tried to distinguish between two cases: whether the two loci are completely homoeologous and carry identical alleles, or they are different loci with no com- 
mon alleles. This comparison was possible for ENP, AP and PGM results, and goodness-of-fit is rather better with the first hypothesis. Furthermore, in the case of two different loci with specific alleles, the existence of null alleles has to be postulated at Enp and Ap loci, without any evidence of "null phenotypes" in the zymograms.

The genetic results obtained in this study support the hypothesis of an allopolyploid origin of apple genome. We refer here to the taxonomical meaning of this word, i.e. a polyploid containing genomes from two related species, as postulated by Sax (1933), Stebbins (1950) and Challice (1981). The existence of two homoeologous genomes, with pairs of loci carrying homoeoalleles and assorting independently in meiosis can explain the bigenic disomic inheritance pattern of most enzymes.

Two cases of monogenic inheritance were found (Est 1 and $I d h)$; this is not incompatible with an allopolyploid hypothesis. Apple is certainly a very ancient polyploid, originating prior to the Cretaceous period (Challice and Westwood 1973). Loss of duplicate gene expression may have occurred in the course of evolution, through the fixation of null variants at one of the redundant loci. This phenomenon was extensively studied in tetraploid fish (Ferris and Whitt 1979), and theoretical models of gene silencing at duplicate loci were proposed (Li 1980). It was reported in tetraploid Chenopodium (Wilson et al. 1983) and also at a high rate in wheat, which is comparatively a recent polyploid (Garcia-Olmedo et al. 1977). The evolutionary significance of the observed level of gene-silencing in apple cannot be infered from this limited study.

Previous genetic studies of morphological or agronomical characters are not in contradiction with an allopolyploid origin of apple genome. Very few characters proved to be simply inherited. Some of them are under monogenic control, such as red colour of all organs or "pale green lethal factor" (Decourtye 1967); others are governed by a pair of complementary genes as in mildew resistance from Malus zumi (Alston 1976) or burr-knots (Decourtye 1967).

Acknowledgements. We wish to thank Prof. R. Tufigo for reviewing the English manuscript, and Drs. J. C. Sanford (Geneva, New York) and D. de Vienne (Orsay, France) for critically reading it and providing useful comments.

\section{References}

Alston FH (1976) Practical aspects of breeding for mildew (Podosphaera leucotricha) resistance in apples. In: Proc Eucarpia Meeting Tree Fruit Breeding. PUDOC, Wageningen, pp 4-17

Cadic A (1982) Pollinisation et incompatibilité interspécifique dans le genre Berberis. Sel Fr 30:37-43
Challice JS (1981) Chemotaxonomic studies in the family Rosaceae and the evolutionary origins of the subfamily Maloideae. Preslia 53:289-304

Challice JS, Westwood MN (1973) Numerical taxonomic studies of the genus Pyrus using both chemical and botanical characters. Bot J Linn Soc 67: 121-148

Chevreau E (1984) Contribution à l'étude de la génétique du Pommier: apport de l'analyse enzymatique. Thèse de Docteur Ingénieur, Orsay

Darlington CD, Moffett AA (1930) Primary and secondary chromosome balance in Pyrus. J Genet 22:129-151

Decourtye L (1967) Etude de quelques caractères à contrôle génétique simple chez le Pommier (Malus sp.) et le Poirier (Pyrus communis). Ann Amelior Plant 17:243-265

De Wet JMJ (1980) Origins of polyploids. In: Lewis WH (ed) Polyploidy: biological relevance. Plenum Press, New York, pp 3-16

Ferris DS, Whitt GS (1979) Evolution of the differential regulation of duplicates genes after polyploidization. J Mol Evol 12:267-317

Garcia-Olmedo F, Carbonero P, Aragoncillo C, Salcedo G (1977) Loss of redundant gene expression after polyploidization in plants. Experientia 34:332-333

Hutchinson ES. Hakim-Elahi A, Miller RD, Allard RW (1983) The genetics of diploidized tetraploid Avena barbata. J Hered 74:381-383

Lespinasse Y (1973) Chromosomic observations in Malus and Pyrus. In: Proc Eucarpia Fruit Sect Symp V. Canterbury, pp 75-83

Lewis EJ. Humphreys MW, Caton MP (1980) Disomic inheritance in Festuca arundinacea Schreb. Z Pflanzenzücht 84: $335-341$

Li WH (1980) Rate of silencing at duplicate loci: a theoretical study and interpretation of data from tetraploid fishes. Genetics 95:237-258

Misic P. Vinterhalter D, Todorovic R (1981) The inheritance of isoperoxidases in apples. Jugosl Vocarstvo 14:43-47

Mulcahy DL (1979) The rise of the angiosperms: a genecological factor. Science 206:20-23

Quiros CF (1982) Tetrasomic segregation for multiple alleles in alfalfa. Genetics 101:117-127

Sanford JC (1983) Ploidy manipulations. In: Moore JN, Janick J (eds) Methods in fruit breeding. Purdue University Press. West Lafayette Ind, pp 100-123

Sax K (1931) The origin and relationships of the Pomoideae. J Arnold Arbor 12:3-22

Sax K (1933) The origin of the Pomoideae. J Am Soc Hortic Sci 30: $147-150$

Stebbins G (1950) Polyploidy and the origin of higher categories. In: Variation and evolution in plants. Columbia University Press, New York, pp 359-362

Vinterhalter DV, James DT (1982/83) The use of peroxidase polymorphism in the identification of apple scion cultivars. Sci Hortic 18:253-261

Wilson HD, Barber SC, Walters T (1983) Loss of duplicate gene expression in tetraploid Chenopodium. Biochem Syst Ecol 11:7-13

Zamir D (1983) Pollen gene expression and selection: applications in plant breeding. In: Tanksley SD, Orton TJ (eds) Isozymes in plant genetics and breeding, part A. Elsevier, Amsterdam, pp 313-330 